\title{
lon sources and low energy beam transport for the new superconducting injector linac for COSY/Jülich
}

\author{
R. Gebel, ${ }^{\text {a) }}$ O. Felden, and P. von Rossen \\ Institut für Kernphysik (IKP), Forschungszentrum, Jülich 52428, Germany
}

(Presented on 13 September 2003; published 17 May 2004)

\begin{abstract}
The Institut für Kernphysik of the Forschungszentrum Jülich $\mathrm{GmbH}$ has been designing and developing a new injector for the cooler synchrotron COSY. To reach the set goal, the projected linac injector will have to be equipped with new ion sources. One ion source will deliver unpolarized; the other polarized $\mathrm{H}^{-}$or $\mathrm{D}^{-}$pulsed beams in the $\mathrm{mA}$ range having an energy of 25 $\mathrm{keV}$. Pulses of up to $500 \mu \mathrm{s}$ in width and repetition rates up to $2 \mathrm{~Hz}$ are foreseen. Several arrangements of the ion sources and the low energy beam transport system have been investigated. The principles guiding the layout of the low energy beam transport are discussed. First operational results of the source for unpolarized $\mathrm{H}^{-}$and $\mathrm{D}^{-}$are discussed. (C) 2004 American Institute of Physics. [DOI: 10.1063/1.1702113]
\end{abstract}

\section{INTRODUCTION}

The future scientific potential of the COSY accelerator facility ${ }^{1}$ could be significantly enhanced if the intensity in the synchrotron could be raised to about $2 \times 10^{11}$ stored polarized protons, which corresponds roughly to the limitation by space charge effects at injection energy. Evaluations by several independent review committees have identified the isochronous cyclotron JULIC, now over 30 years old, as being the bottleneck dwarfing attempts to reach this goal. As the injection efficiency into COSY is limited by emittance growth through the stripper foil and tune shift, it does not help to increase the injection time beyond its present value of $20 \mathrm{~ms}$. Alternatively raising the beam intensity of JULIC to the required level is not feasible. Studies showed that the most suitable new injector for COSY would be a superconducting linac with independently phased single-cell cavities (COSY) space-charge limit (SCL). ${ }^{2,3}$ Such an injector would be capable of filling COSY for polarized protons and deuterons at the chosen injection energy of about $50 \mathrm{MeV}$. For instance, a short pulsed beam of $0.5 \mathrm{~mA}$ for $200 \mu$ s provides $5 \times 10^{11}$ particles for stripping and would at the same time significantly reduce the emittance growth of the beam.

To keep civil engineering costs low, the new injector had to fit in the existing buildings. Nonetheless, it was necessary to locate the ion source complex in a small rather inexpensive annex to the building to provide enough room for the accelerating structures. Figure 1 shows the layout of the COSY SCL. In addition the assembly and commissioning of the new injector should not interfere with the ongoing experimental program at COSY. The performance of the ion sources is a crucial element in reaching the required specifications. To keep downtime as low as possible for an accelerator that runs over $7000 \mathrm{~h} / \mathrm{year}$, and to enhance flexibility, two ion sources will be installed. One ion source will deliver unpolarized the other source will deliver polarized $\mathrm{H}^{-}$and $\mathrm{D}^{-}$beams with an energy of $25 \mathrm{keV}$ and pulses of up to 500

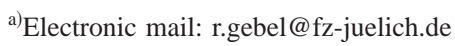

$\mu$ s width at a maximum repetition rate of $2 \mathrm{~Hz}$. For providing unpolarized beams with a commercial multicusp source, a 4/3 $\mathrm{N}$ ion source from the company IBA was chosen, and has already been delivered. It has been foreseen to use this source for running the accelerator while construction of the polarized source was still in progress.

\section{FIRST ACCELERATION STAGE}

The first acceleration occurs in a radio frequency quadrupole (RFQ) ${ }^{4}$ bringing the ions to $2.5 \mathrm{MeV} / \mathrm{u}$. It also bunches the beam and shapes the phase space according to the requirements of the super conducting linac. For each ion species a separate RFQ is necessary, due to the different velocity profiles. A mechanical rail system with Einzel lenses, RFQs, and booster cavities will allow switching the ion species in less than a working day. The RFQs and the short booster cavities have been designed in collaboration with the University of Frankfurt/Main. Einzel lenses and RFQs are designed for an input energy of $25 \mathrm{keV}$.

The ion sources are mounted in such a way that the polarized source can be tuned and tested even when the injector is operated with unpolarized beams. A Wien filter or alternatively a solenoid is included in the low energy beam transport (LEBT) to properly align the spin axis. The LEBT beam line will be kept as short as possible to reduce blowup of the beam due to space-charge effects, otherwise much larger beam envelopes would have to be accepted. The ion optical elements must be adjustable over a wide range of magnetic rigidities.

\section{ION SOURCE DESIGN CONSIDERATIONS}

The new ion sources have to meet certain specifications with respect to peak intensity, pulse length, and achievable polarization in the case of the polarized ion source. Our laboratory has a long-standing experience with polarized ion sources. It operates a colliding beams ion source ${ }^{5}$ where the charge transfer to the neutral nuclear polarized hydrogen is being done with a pulsed neutral $50 \mathrm{keV}$ Cs beam. This 


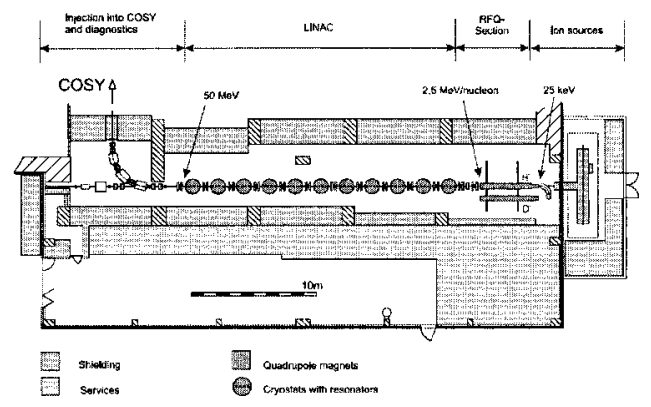

FIG. 1. Layout of the proposed superconducting injector LINAC for the cooler synchrotron COSY (COSY SCL). Detailed view of the low energy part of the injector linac area with the ion source section, RFQ section, the superconducting linac section, and diagnostics.

source can deliver pulses with a length of $25 \mathrm{~ms}$ but is limited in peak intensity to about $50 \mu \mathrm{A}$. The present polarized ion source, because of its operation principle, cannot fulfill the required demands. As the stripping injection scheme for COSY will be kept $\mathrm{H}^{-}$and $\mathrm{D}^{-}$beams have to be provided.

The new ion sources are expected to deliver beam currents of up to $2 \mathrm{~mA}$ but for a much shorter time compared with the present situation. It is envisioned to accelerate pulses lasting up to $500 \mu$ s that would fill COSY well to its space-charge limit. The $2 \mathrm{~mA}$ peak current limitation is a result of the available rf power of the solid state amplifiers that feed the superconducting cavities. It is, therefore, essential for the performance of the new ion sources that they can deliver as many ions as possible in a short time and within the acceptance of the injector. The repetition rate of these pulses can be raised to $2 \mathrm{~Hz}$ for tuning, but it will be much lower in the standard operational mode.

\section{UNPOLARIZED ION SOURCE}

For the present injector a specialized source of negative ions for cyclotrons is used. The IBA series $4 / 3 \mathrm{~N}$ ion source comprises an arc discharge chamber, the "ion source," and a low energy triode accelerator with an integrated electron trap. One 4/3 N ion source from AEA Culham has been in operation at the cyclotron JULIC since $1997 \mathrm{in} \mathrm{cw}$ and pulsed operation. Pulsing is performed either by switching the puller voltage or the chamber voltage. The required modes of operation for JULIC and for the new injector and the properties of the source have been investigated. The ion source is also used for commissioning ion optical elements like Einzel lenses, steerer, and dipole magnets and electrostatic prisms. In addition beam diagnostic elements like current pickups, cups, scanner, and viewer are tested to provide the needed tools to operate the source in the COSY environment. The source has been slightly modified in our laboratory taking advantage of the experience gained with the existing source. The new ion source demonstrated the required performance for $\mathrm{H}^{-}$. Figure 2 depicts data collected during source tuning under varying extraction conditions.

As an additional option a pulsed mode of operation of the ion sources was investigated. The $4 / 3 \mathrm{~N}$ ion sources are equipped with an accel-decel extraction electrode configuration. The electrode close to the extraction aperture is called a puller. Using a push-pull transistor switch capable of

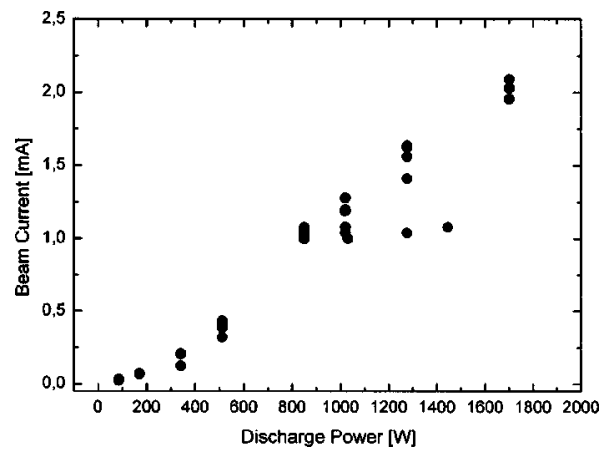

FIG. 2. The beam current measured on a compensated cup positioned 1.25 $m$ downstream of the LEBT, displayed as a function of discharge power.

switching in short times and to withstand high voltages, the puller is switched between source potential and extraction voltage. A nondestructive current monitor (Pearson 110) measures the beam current. Figure 3 shows the result of one measurement sequence. The puller is switched on five times for a duration of $200 \mu$ s with a duty factor of $50 \%$.

\section{POLARIZED ION SOURCE}

For the delivery of short pulses of high intensity polarized ions at $25 \mathrm{keV}$ to the RFQ, a CIPIOS $^{6}$ type ion source has been foreseen. This kind of source had been in routine operation for several years at the Indiana University Cyclotron Facility (IUCF). There, its operational characteristics had matured over time. In collaboration with V. Derenchuk (IUCF) and A. Belov (INR), the performance of the source has been improved, both in pulse length and intensity. ${ }^{7}$ With a modified pulsed plasma source and a new neutralizerconverter setup, an intensity of $1.5 \mathrm{~mA}$ (peak) and a pulse width of over $400 \mu$ s have been achieved. It was envisioned to either to acquire the IUCF source or to build a source of this type in Jülich, named ISPOLIN.

\section{LEBT SECTION WITH SPACE CHARGE AND PHASE SPACE MATCHING TO THE RFQ}

The beams from the two ion sources have to be transported to the RFQ section with minimal losses, especially for polarized particles. When using solenoids one has to keep in mind that they rotate the spin vector with respect to the longitudinal field axis. A concept with oppositely connected pairs of solenoids compensates this spin rotation. Also angular kicks for ions are nearly compensated. The projected

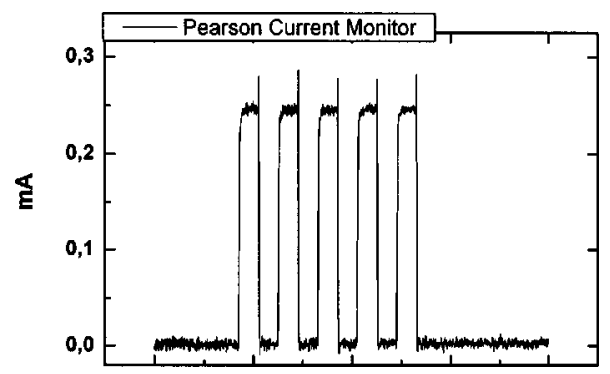

FIG. 3. Example for beam pulsing with high voltage switch at the puller electrode. The extracted beam current is monitored by a current transformer in the beam line to the injector. 

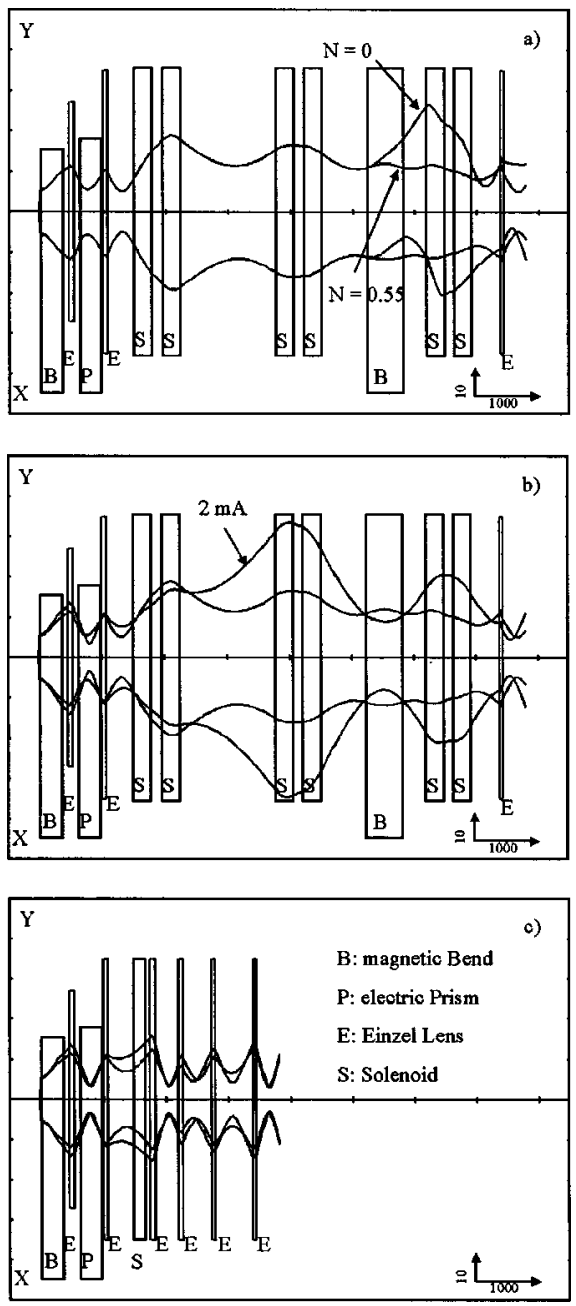

FIG. 4. Transport of the polarized beam from the ion source extraction (left) to the entrance of the RFQ (right) at $25 \mathrm{keV}$. (a) The envelopes of a low intensity beam are compared to those of a $2 \mathrm{~mA}$ beam with space charge. (b) The $90^{\circ}$ bending magnet with field index of 0.55 produces a narrow envelope with a double waist at the RFQ entrance. The result of a zero field index is also shown for comparison. (c) LEBT without $90^{\circ}$ bending magnet and electrostatic elements. The transverse full scale is $70 \mathrm{~mm}$ for $x$ and $y$. The full distance over the horizontal scale is $9.0 \mathrm{~m}$.

beam properties required a beam pipe with $100 \mathrm{~mm}$ diameter, resulting in a preferred length of the solenoids of about $300 \mathrm{~mm}$ to keep the effect of fringe fields on the particle motion and spin rotation low. A $150 \mathrm{~mm}$ distance between the solenoids avoids overlap of the fringe fields. The layout of the LEBT is depicted in Fig. 4. A section for spin handling with a Wien filter or a single solenoid is foreseen. The beam line for the unpolarized beam is designed in analogy. Due to the drift space between the first and second solenoid pair, a space left for future spin manipulation by a Wien filter, and through the following $90^{\circ}$ bend, the uncompensated beam reaches the limits of the beam tube for a beam current of 2 mA, as shown in Fig. 4(a). Beam loss would occur for higher currents. This result is confirmed by experience with the transport of the unpolarized beam from the CIPIOS source at IUCF. Symmetric envelopes in transverse directions and a double waist at the entrance of the RFQ can be achieved by using a $90^{\circ}$ bending magnet without pole-face rotation but having a field index of $n=0.55$, as shown in Fig. 4(b).

\section{ALTERNATIVE LEBT SYSTEM}

The preferable and compelling solution would be a straight LEBT system without a $90^{\circ}$ bending magnet for the polarized beams, if additional floor space would become available by appropriate changes to the building. During the design process the position of the ion sources and the layout of the LEBT system was modified. The latter is much shorter now and more similar to the one used at IUCF. The foreseen much shorter layout of the LEBT using only electrostatic lenses for focusing the beam seems to be preferable and is depicted in Fig. 4(c). Measurements at IUCF showed that such a system is capable of achieving good transmission for the expected quality and intensity of the beams. This type of beam line has been successfully used at IUCF together with the CIPIOS polarized ion source and a similar configuration could be implemented in our case for connecting the polarized ion source and RFQ. A solenoid allows the spin preparation for protons and deuterons. The beam is focused by electrostatic lenses and kept axially symmetric; the spin direction is left unaffected. The short length of this beam line reduces the effects of space charge during beam transport, which is an important consideration in view of beam currents in the $\mathrm{mA}$ range. The results of TRANSPORT calculations shown in Fig. 4(c) compare the beam behavior with and without space-charge effects.

\section{ACTUAL STATUS}

At the very end of the year 2002 due to unprecedented budget cuts imposed by the federal government the Forschungszentrum Jülich was left with no other choice but to suspend the COSY SCL project for at least 2 years. However, some prototype work is still underway and first results can be expected during 2003/2004. This setback due to money shortage has not altered the conviction that this new linac will be an important scientific addition to the COSY accelerator facility.

\section{ACKNOWLEDGMENTS}

The authors are deeply indebted to V. Derenchuk and A. $\mathrm{S}$. Belov for their support and involvement as well as providing the opportunity to make studies at the CIPIOS source at IUCF. This work is partly funded by the European Community under Contract No. HPRI-CT-2001-50021.

\footnotetext{
${ }^{1}$ R. Maier, Nucl. Instrum. Methods Phys. Res. A 390, 1 (1997).

${ }^{2}$ COSY-SCL Conceptual Design Report, Internal Report, Forschungszentrum Jülich, October 2001 edited by H. Jungwirth 〈http://www.fzjuelich.de/ikp/linac-public/>

${ }^{3}$ The Superconducting Linac for the Cooler Synchrotron COSY, Design Update March 2002, Internal Report, Forschungszentrum Jülich, edited by H. Jungwirth.

${ }^{4}$ K. U. Kuehnel et al., Proceedings of the 2003 Particle Accelerator Conference, Portland, OR, 2003, RPAB041.

${ }^{5} \mathrm{O}$. Felden et al., Proceedings of the 9th International Workshop on Polarized Sources and Targets, Nashville, TN, 2002, p. 200.

${ }^{6}$ V. P. Derenchuk et al., Proceedings of the 2001 Particle Accelerator Conference, Chicago, 2001, p. 2093.

${ }^{7}$ V. P. Derenchuk et al., Proceedings of the 9th International Workshop on Polarized Sources and Targets, Nashville, 2002, p. 210.
} 